\title{
Estrutura populacional de Eschweilera coriacea em floresta de várzea no município de Afuá, Pará
}

\author{
Bruno Rafael Silva de Almeida ${ }^{1}$ Bruno de Almeida Lima ${ }^{2}$ João Ricardo Vasconcellos Gama ${ }^{2}$ Andrea Araújo \\ da Silva ${ }^{2}$ Renato Bezerra da Silva Ribeiro ${ }^{2}$ Girlene da Silva Cruz ${ }^{1}$ Daniele Lima da Costa ${ }^{3}$ Misael Freitas \\ dos Santos $^{3}$
}

${ }^{1}$ Universidade de Santa Catarina, Av. Luiz de Camões, 2090 - Conta Dinheiro, Lages - SC, 88520-000
${ }^{2}$ Universidade Federal do Oeste do Pará, Rua Vera Paz, s/n (Unidade Tapajós) Bairro Salé, Santarém-PA, 68040-255
${ }^{3}$ Universidade Estadual do Centro Oeste, Rodovia PR 153, km 7, Riozinho, Irati-PR, 84500-000

*Author for correspondence: almeida.bruno.r.s@gmail.com

Received: September 2018 / Accepted: March 2019 / Published: June 2019

\section{Resumo}

O objetivo do presente estudo foi analisar a estrutura populacional da espécie Eschweilera coriacea (DC.) S.A Mori em duas áreas de várzea no município de Afuá, Pará. Foram alocadas, sistematicamente, 29 unidades amostrais de $20 \mathrm{~m}$ x $250 \mathrm{~m}$ em floresta de várzea alta e 25 unidades amostrais de $20 \mathrm{~m}$ x $250 \mathrm{~m}$ em floresta de várzea baixa, onde foram mensuradas as árvores com diâmetro medido à $1,30 \mathrm{~m}$ do solo $(\mathrm{DAP}) \geq 15,0 \mathrm{~cm}$. Cada unidade amostral continha uma subunidade de $100 \mathrm{~m}^{2}$, onde foram inventariadas todas as plantas com altura $\geq 0,3 \mathrm{~m}$ e DAP $<$ $15,0 \mathrm{~cm}$. Considerando DAP $\geq 10 \mathrm{~cm}$, ocorreram 26,69 árv.ha ${ }^{-1}$ na várzea alta e 23,61 árv.ha ${ }^{-1}$ para várzea baixa,

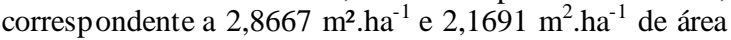
basal, e volumetria de $31,6740 \mathrm{~m}^{3} \cdot \mathrm{ha}^{-1}$ e $22,6689 \mathrm{~m}^{3} \cdot \mathrm{ha}^{-1}$, respectivamente. $\mathrm{O}$ padrão de distribuição espacial em ambos ecossistemas foi agrupado. No estrato de regeneração natural (altura $\geq 0,3 \mathrm{~m}$ e $\operatorname{DAP}<10,0 \mathrm{~cm}$ ) foram inventariadas 71 plantas na várzea alta $(244,83$ árv.ha $\left.{ }^{-1}\right)$ e 68 indivíduos na várzea baixa $\left(272,00\right.$ árv.ha $\left.{ }^{-1}\right)$. A comparação demonstrou que as populações de várzea alta e várzea baixa são distintas.

Palavras-chave: Inventário Florestal, Ecologia, Amazônia

\begin{abstract}
The aim of the present study was to analy ze the population structure of Eschweilera coriacea S.A Mori in two areas of floodplain situated in the municipality of Afuá, Pará. It was allocated sy stematically 29 sample plots of $20 \mathrm{mx} 250$ $\mathrm{m}$ (14.5 ha) in the high floodplain and 25 plots of $20 \mathrm{~m} \mathrm{x}$ $250 \mathrm{~m}(12.5 \mathrm{ha})$ in the low floodplain forest. All individual trees with diameter at breast height $(\mathrm{DBH}) \geq 15 \mathrm{~cm}$ were measured. Each sample contained a sub plot of $100 \mathrm{~m}^{2}$ for natural regeneration analysis, where all individuals with height $\geq 0.3 \mathrm{~m}$ and $\mathrm{DBH}<15.0 \mathrm{~cm}$ were inventoried. Considering DHB $\geq 10 \mathrm{~cm}$, occurred 26.69 trees $^{-1} \mathrm{ha}^{-1}$ in the high floodplain and 23.61 trees.ha $^{-1}$ in the low floodplain, corresponding to $2.8667 \mathrm{~m}^{2} \cdot \mathrm{ha}^{-1}$ and $2.1641 \mathrm{~m}^{2} \cdot \mathrm{ha}^{-1}$ of basal area, and volumetry of $31.6740 \mathrm{~m}^{3} \cdot \mathrm{ha}^{-1}$ and 22.6689 $\mathrm{m}^{3} \cdot \mathrm{ha}^{-1}$, respectively. The spatial distribution pattern for both ecosystems was grouped. In the natural regeneration stratum, 71 plants were recorded for high floodplain (244.83 trees.ha $\left.{ }^{-1}\right)$ and 68 plants for low floodplain (272.00 trees.ha ${ }^{-1}$ ). The comparison showed that the populations of high lowland and low floodplain are distinct.
\end{abstract}

Keyword: Forest Inventory, Ecology, Matamata.
A várzea é um dos ecossistemas amazônicos de grande importância ecológica e socioeconômica, onde populações ribeirinhas praticam a pesca, agricultura, extrativismo de produtos madeireiros e não madeireiros (Gama et al. 2003). Periodicamente, a vegetação de várzea é alagada por rios de água branca, que dominam boa parte da planície amazônica (Ferreira et al. 2005). No estuário Amazônico ocorre a várzea flúvio-marinha, que em termos estruturais e funcionais é dividida em várzea baixa - alagada durante o inverno amazônico (fevereiro a junho), e várzea alta - que inunda nas lançantes de maré e durante as chuvas intermitentes de inverno (Lima et al. 2000).

Por causa do dinamismo hidrológico e geomorfológico dos rios, há uma diferença de idade, fisionomia e composição nas florestas de várzea em várias comunidades (Wittmann e Junk 2003). Na Amazônia brasileira a floresta de várzea representa $75.880,8 \mathrm{~km}^{2}$, isto é, 1,6\% da superfície (Macedo et al. 2007). A vegetação desses ambientes apresenta diferentes composições florísticas, com um expressivo número de espécies de palmeiras, espécies arbóreas de importância comercial e espécies que apresentam potencial alimentar e farmacêutico (Queiroz 2008; Renó et al. 2011).

Analisar a estrutura das florestas corresponde a primeira etapa para o conhecimento do potencial econômico da vegetação (Bentes Gama et al. 2002a). Por meio da análise estrutural se obtém informações sobre autoecologia, possibilidades de aproveitamento, potencial produtivo e estádio sucessional que são importantes para o planejamento do manejo florestal e práticas silviculturais (Higuchi et al. 1985; Seitz 1988).

A espécie Eschweilera coriacea (DC.) S.A Mori, da família Lecythidaceae, pertence ao grupo ecológico das espécies clímax exigente de luz, ocorrente em toda região amazônica. É uma árvore de grande porte, podendo alcançar altura de até 35 metros e diâmetro de até $90 \mathrm{~cm}$, sua madeira é resistente e durável e propicia para a construção civil (esteio, pernamancas, ripas, tábuas, cabos de ferramentas e marcenaria em geral) e produção de energia (Mori e Prance 1990; Gama et al 2002; Lorenzi 2009; Almeida 2010). Além disso, a espécie apresenta potencial biotecnológico, pois o seu extrato vegetal pode atuar como agente antioxidante e inibidor do crescimento de organismos patogênicos (Freitas et al. 2005).

Vale destacar, que apesar da importância da espécie no setor da construção civil, ainda são escassos estudos referentes a estrutura populacional da espécie em ambientes de várzea, sendo observados alguns estudos sobre sua fitossociologia apenas em ecossistema de terra firme.

Introdução 
Diante desse contexto, o estudo foi desenvolvido com o objetivo de analisar a estrutura populacional da espécie Eschweilera coriacea S.A M ori em duas áreas de várzea no município de Afuá, Pará.

\section{Material e métodos}

Área de estudo

O estudo foi realizado na propriedade florestal da Exportadora de Madeiras do Pará Ltda. (EMAPA), localizada no município de Afuá $\left(0^{\circ} 09^{\prime} 24\right.$ " S e $50^{\circ} 23^{\prime}$ 12 " W), estado do Pará. A área total do local corresponde à 1.200 ha, na qual 80 ha são de floresta de várzea baixa explorada e 1.120 ha são florestas de várzea alta, dos quais 500 ha foram utilizados em projetos de enriquecimento florestal e os demais 620 ha de floresta não explorada (Gama et al. 2003).

O solo da região de Afuá é representado principalmente pelos solos Gley Eutróficos desenvolvidos sobre sedimentos recentes do quaternário. Apresentam alta fertilidade, devido à elevada porcentagem de matéria orgânica depositada por causa da movimentação das marés. A precipitação anual é sempre maior que 2.500 $\mathrm{mm}$, apresenta umidade relativa do ar em torno de $80 \%$ e a temperatura média de $26^{\circ} \mathrm{C}$, com mínima de $18^{\circ} \mathrm{C}$ e máxima de $36^{\circ} \mathrm{C}$. A vegetação predominante da região são as florestas de várzea periodicamente alagadas, apresentando cobertura arbórea com espécies de alto valor econômico e formações com espécies herbáceas e palmeiras (Barbosa et al. 2012).

\section{Amostragem e coleta dos dados}

Foram distribuídas sistematicamente 29 unidades amostrais de $20 \mathrm{~m}$ x $250 \mathrm{~m}$ para várzea alta (VA) e 25 unidades amostrais de $20 \mathrm{~m}$ x $250 \mathrm{~m}$ para várzea baixa (VB). Em cada unidade amostral foram mensuradas as árvores com diâmetro medido à $1,30 \mathrm{~m}$ do solo (DAP) $\geq$ $15,0 \mathrm{~cm}$, nestas foram registradas as informações de DAP, altura total (h) e altura comercial (hc). Na subparcela de 10 $\mathrm{m} \times 10 \mathrm{~m}\left(100 \mathrm{~m}^{2}\right)$, onde foram inventariadas todas as plantas com altura $\geq 0,3 \mathrm{~m}$ e DAP $<15,0 \mathrm{~cm}$. Em cada subparcela foram coletadas informações referentes ao DAP e altura total.

O nome regional da espécie foi registrado em campo, por identificador experiente. O material botânico foi coletado e posteriormente identificado por especialistas do Laboratório de Botânica da EMBRAPA Amazônia Oriental, em Belém, PA. Para conferência da grafia foi consultado o banco de dados do Reflora, disponível na página

http://floradobrasil.jbrj.gov.br/jabot/floradobrasil/FB1796 5.

\section{Análise de dados}

As plantas foram divididas em duas classes de tamanho, a saber: estrato adulto (DAP $\geq 10 \mathrm{~cm}$ ) e regeneração natural (altura $\geq 0,3 \mathrm{~m}$ a DAP $<10 \mathrm{~cm}$ ). $\mathrm{O}$ estrato adulto foi analisado por meio dos parâmetros fitossociológicos de densidade e dominância estimados segundo Mueller-Dombois e Ellenberg (1974). Para a análise de distribuição espacial usou-se o Índice de Morisita (IMi), conforme a recomendação de Brower e Zar (1977):

onde:

$$
I M_{i}=\frac{n \cdot\left(\sum x^{2}-N\right)}{N \cdot(N-1)}
$$

$I M_{i}$ - índice de Morisita; $n$ - número de parcelas amostradas; $N$ - número total de árvores, considerando as $n$ parcelas; e, $x^{2}$ - quadrado do número de árvores por parcela.

A significância do Índice de Morisita foi obtida pelo teste de Qui-quadrado a 5\% de probabilidade:

onde:

$$
X^{2}=\frac{n \cdot\left(\sum x^{2}\right)}{N}-N
$$

$X^{2}$ - estatística para o teste Qui-quadrado a 5\% de probabilidade; $n$ - número de parcelas amostradas; $N$ número total de árvores, considerando as $\mathrm{n}$ parcelas; $x^{2}$ quadrado do número de árvores por parcela.

Os parâmetros estruturais da regeneração natural foram estimados de acordo com Curtis e Mcintosh (1951). Para avaliação da estrutura vertical da regeneração natural, utilizou-se as classes de tamanho (CT) sugeridas pela FAO (1971), sendo: CT1: 0,3 m $\leq \mathrm{h}<1,5 \mathrm{~m} ; \mathrm{CT} 2: 1,5 \mathrm{~m} \leq \mathrm{h}$ < 3,0 m; CT3: h 3,0 m $\geq$ até DAP < 5,0 cm e; CT4: 5,0 $\mathrm{cm} \leq \mathrm{DAP}<10,0 \mathrm{~cm}$. Em que: h corresponde à altura total e DAP ao diâmetro a $1,30 \mathrm{~m}$ do solo, em $\mathrm{cm}$.

As árvores com DAP $\geq 10 \mathrm{~cm}$ foram distribuídas em oito classes diamétrica com intervalo de $10 \mathrm{~cm}$. A distribuição volumétrica foi analisada, sendo o volume calculado da seguinte forma:

$$
v_{c i}=\frac{\pi}{40.000} D A P^{2} \cdot h_{c} \cdot 0,7
$$

em que: $v=$ volume comercial de madeira, em $\mathrm{m}^{3} ; D A P=$ diâmetro a 1,30 m do solo, em $\mathrm{cm} ; h_{c}=$ altura comercial, em m; 0,7 = fator de forma de Heinsdijk e Bastos (1963).

Para a comparação de variáveis estruturais entre as florestas de várzea alta e várzea baixa, utilizou-se dois tratamentos, sendo: T1 - várzea alta e T2 - várzea baixa. As variáveis foram analisadas pelo teste t, de Student, para amostras independentes, para testar a existência de igualdade entre as médias dos parâmetros estudados, densidade, área basal e volume, a 5\% de probabilidade. Foram aplicados testes prévios de normalidade e homogeneidade, a normalidade foi verificada pelo teste de Shapiro-Wilk a 5\% de probabilidade, quando necessário aplicou-se transformação logarítmica e quadrática. Os dados que não atingiram normalidade foram analisados por meio da estatística não paramétrica pelo Teste de Mann-Whitney a 5\% de probabilidade, a homogeneidade foi verificada pelo teste $\mathrm{F}$ a $5 \%$ de probabilidade. A tabulação e o processamento de dados foram realizados por meio da planilha eletrônica Excel 2016 e o software BioEstat 5.0.

\section{Resultados}

\section{Estrutura da população}

Foram registradas 683 árvores da espécie E. coriacea, sendo 387 na várzea alta (VA) e 296 na várzea baixa (VB), correspondendo a uma densidade de 26,69 árv.ha${ }^{1}$ e 23,61 árv.ha ${ }^{-1}$, respectivamente. A densidade diferiu estatisticamente entre os ecossistemas ( $p>0,05)$. Quanto a dominância, na VA foi de $2,8667 \mathrm{~m}^{2}$.ha ${ }^{-1}$, enquanto que na VB foi igual a $2,1691 \mathrm{~m}^{2} \cdot \mathrm{ha}^{-1}$, valores estes que se diferenciaram estatisticamente pelo teste não paramétrico de Mann-Whitney em nível de 5\% de probabilidade $(\mathrm{p}>0,05)$.

As distribuições diamétricas da espécie em VA e VB apresentaram maior concentração de árvores nas classes intermediárias e uma menor frequência nas maiores classes (Figura 1), não apresentando o padrão de " $J$-invertido" característico de florestas nativas. A classe de $35 \mathrm{~cm}$ 
apresentou as maiores densidades para os ambientes, sendo que a várzea alta ap resentou densidade de 7,52 árv.ha ${ }^{-1}$ e várzea baixa densidade de 7,2 árv.ha ${ }^{-1}$.

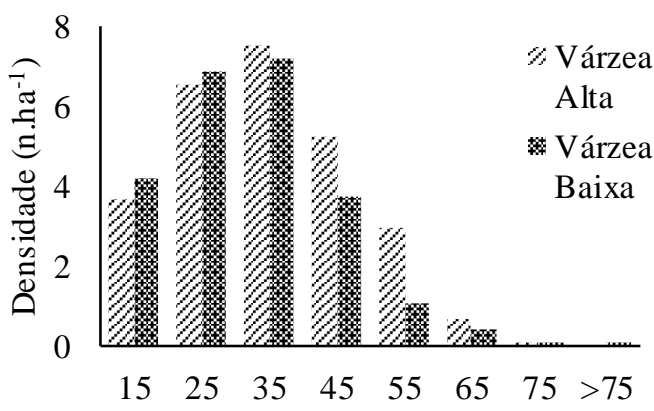

Centro de classe de DAP $(\mathrm{cm})$

Figura 1. Distribuição diamétrica da Eschweilera coriacea S.A M ori, propriedade florestal da EM APA, município de Afuá, Pará.

O volume comercial da E. coriacea foi 31,6740 $\mathrm{m}^{3} \cdot \mathrm{ha}^{-1}$ e $22,6689 \mathrm{~m}^{3} \cdot \mathrm{ha}^{-1}$ para VA e VB respectivamente, demostrando que a floresta de várzea alta estava mais estocada que a de várzea baixa, o que se deve provavelmente à exploração florestal madeireira que ocorreu em várzea baixa. Na figura 2, verifica-se uma distribuição em forma de sino para ambas as várzeas. A partir do centro de classe $65 \mathrm{~cm}$, em específico, o decréscimo do volume está relacionado com a baixa densidade de indivíduos presentes nesta classe de diâmetro.

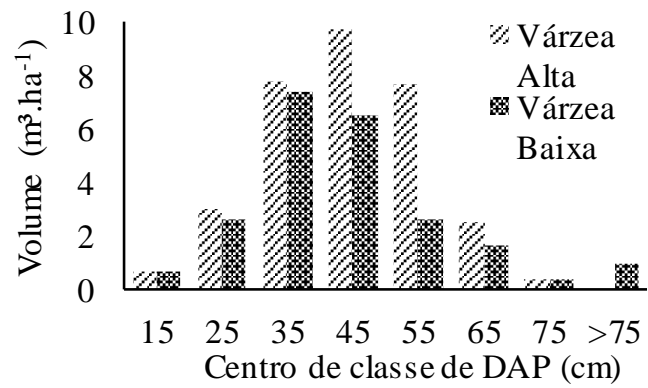

Figura 2. Distribuição volumétrica por classe de diâmetro Eschweilera coriacea S.A Mori, propriedade florestal da EMAPA, município de Afuá, Pará.

No estrato de regeneração natural foram registradas 139 plantas da espécie E. coriacea, sendo 71 plantas em VA $\left(244,83\right.$ árv.ha $\left.^{-1}\right)$ e 68 plantas para VB $\left(272,00\right.$ árv.ha $^{-}$ ${ }^{1}$ ), em relação a distribuição das plantas por classe de tamanho, observou-se distribuição com tendência ao formato de " $J$-invertido" na primeiras classes de tamanho, sendo $52,11 \%$ das plantas na primeira classe de tamanho (CT1), 21,13\% na classe de tamanho 2, 12,68\% na classe de tamanho 3 e $14,08 \%$ na classe de tamanho 4, para a VA. E para a VB, 33,82\% dos indivíduos na classe de tamanho $1,29,41 \%$ na classe de tamanho $2,10,29 \%$ na classe de tamanho 3 e 26,49\% na classe de tamanho 4 (Figura 3).

Em relação aos parâmetros qualitativos, verificou-se que a qualidade da regeneração natural foi satisfatória, pois na VA e VB $92 \%$ e $85,14 \%$, respectivamente, apresentaram-se vivos e eretos. Os indivíduos que apresentaram algum tipo de danos ou caídos foram $8,00 \%$ e $14,86 \%$ para VA e VB, respectivamente.

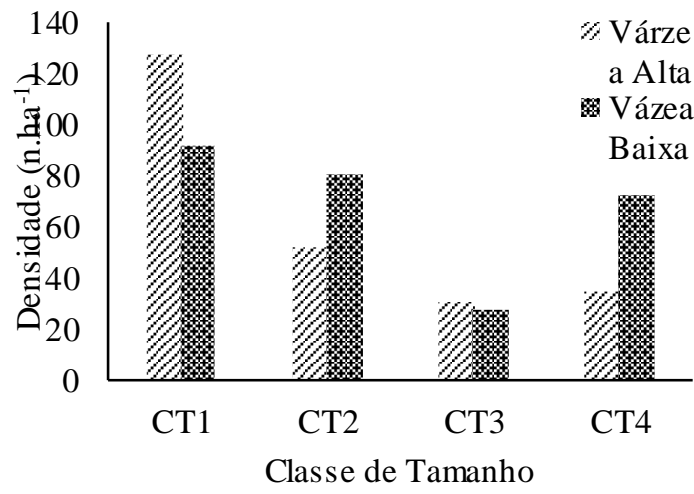

Figura 3. Distribuição das classes de tamanho de Eschweilera coriacea S.A Mori, propriedade florestal da EMAPA, município de Afuá, Pará.

A distribuição espacial tanto na VA como na VB, o índice de Morisita representou um padrão agregado para a espécie, sendo 1,07 e 1,26, respectivamente. A distribuição da espécie nas classes diâmetro indicam que a espécie é pertencente ao grupo das clímax exigente de luz por apresentar a concentração de plantas nas classes intermediarias.

\section{Discussão}

Ao comparar a estrutura populacional da espécie, foi verificado que Almeida et al. (2012) ao realizarem um inventário (DAP $\geq 10,0 \mathrm{~cm}$ ) em floresta de terra firme no município de Santarém, na região Oeste do Pará, identificaram E. coriacea entre as arbóreas de maior importância ecológica, ocorrendo com 23,75 árv.ha ${ }^{-1}$. Silva-Ribeiro et al. (2013) ao mensurarem espécies, na mesma região, $\operatorname{com} \mathrm{DAP} \geq 10,0 \mathrm{~cm}$ identificaram a $E$. coriacea entre as de maior densidade absoluta, mais de nove árvores por hectare.

Aparício (2011) ao inventariar árvores com DAP $\geq$ $10,0 \mathrm{~cm}$ em floresta de terra firme na Resex do rio Cajarí, identificou a espécie com densidade de 29,55 árv.ha ${ }^{-1}$ e dominância de $1,85 \mathrm{~m}^{2} \cdot \mathrm{ha}^{-1}$, sendo considerada a terceira espécie mais importante na área de acordo com o valor de importância. De modo geral, E. coriacea apresenta alta ocorrência em florestas de terra firme no estado do Pará (Gama et al., 2005).

E. coriacea apresentou distribuição diamétrica com forma assimétrica positiva, que pode ser característica da espécie, uma vez que a mesma é classificada como clímax exigente de luz, e os menores indivíduos apresentam dificuldade para se desenvolverem em ambiente sombreado, condições encontradas no estrato inferior da floresta. Além disto, ao analisar estrutura diamétrica de espécies individuais é comum não encontrar distribuição em formato de "j-invertido" (Queiroz, 2004; Costa et al., 2018).

A volumetria está diretamente ligada à distribuição diamétrica e a altura, sendo esse um fator que pode explicar o baixo potencial volumétrico nas primeiras classes. A distribuição volumétrica apresentou o formato de sino, aproximando-se da distribuição normal, em que o volume é semelhante para pontos de mínima e máxima classe de diâmetro (Silva 2006). 
Em relação a análise estrutural da regeneração natural, a maior ocorrência de indivíduos na primeira classe de tamanho também foi registrada no estudo de Jardim e Vieira (2001). Isso demostra bom estado de conservação da floresta, pois a regeneração possivelmente irá repor o estoque de árvores adultas no decorrer dos processos dinâmicos da floresta (Batista e Gonçalves 2013).

Quanto ao padrão de distribuição espacial encontrado para a espécie nos dois ambientes, este pode ser influenciado por fatores abióticos como textura, fertilidade e disponibilidade hídrica do solo, luminosidade e temperatura, e as disponibilidades desses recursos influenciam a distribuição desses indivíduos (Bernasol e Lima-Ribeiro 2010). Além disto a agregação da espécie na área de estudo pode estar relacionada com a longa duração e alta intensidade de exploração, grande ocorrência de fontes de sementes, diferentes níveis de inundação da floresta, entre outros fatores (Gama et al. 2002). Espécies florestais apresentam padrão de distribuição agrupado, quando as mesmas encontram condições favoráveis à sua germinação e a sua sobrevivência (Silva et al. 2011).

\section{Conclusões}

A estrutura da população de Eschweilera coriacea segue as mesmas tendências em várzea alta e baixa, com maior estoque em área de várzea alta não manejada.

\section{Referências}

Almeida AF (2010) Análise etnoecológica da floresta de várzea da ilha de Sororoca, Ananindeua, Pará, Brasil. Dissertação. Universidade Federal do Pará, Belém. 61p.

Almeida LS, Gama JRV, Oliveira FA, Carvalho JOP, Goncalves DCM, Araúio GC (2012) Fitossociologia e uso múltiplo de espécies arbóreas em floresta manejada, comunidade Santo Antônio, município de Santarém, estado do Pará. Acta Amazonica, 42(2):185-194.

Aparício WCS (2011) Estrutura da vegetação em diferentes ambientes na resex do rio Cajari: interações solo-floresta e relações com a produção de castanha. Tese, Universidade Federal Rural de Pernambuco, Recife. 150p.

Barbosa MJS, Carvalho KFC, Cruz WC, Eid F, Félix ODL, Guedes LPF, Santos MAR, Santos RAS, Souza EJL (2012) Relatório analítico do território do Marajó Universidade Federal do Pará, Belém, PA. 79p.

Batista JF, Gonçalves M A (2013) Florística e estrutura da regeneração natural arbórea de uma floresta de várzea na Reserva Extrativista Chocoaré - M ato Grosso, Pará, Brasil. Biota Amazônia, 3(3):139-145.

Bentes-Gama MM, Scolforo JRS, Gama JRV (2002a) Potencial produtivo de madeira e palmito de uma floresta secundária de várzea baixa no Estuário Amazônico. Revista Árvore, 26(3):311-319.

Bernasol WP, Lima-Ribeiro MS(2010) Estrutura espacial e diamétrica de espécies arbóreas e seus condicionantes em um fragmento cerrado sentido restrito no sudoeste goiano. Revista Hoehnea, 37 (2): 181-198.

Brower JE, Zar JH (1977) Field and laboratory methods for general ecology. Dubique: Win. C. Brown Publishers, 2ed. 226p.

Costa DL, Santos MF, Bezerra TG, Silva Ribeiro RB, Gama JRV, Melo LO, Ximenes LC, Coelho AA (2018)
Estrutura e distribuição espacial de Symphonia globulifera L.f. em floresta de várzea baixa, Afuá - PA. Advances in Forestry Science, 5(1): 275-281.

Curtis JT, Mcintosh RP (1951) An upland forest continuum in the prairie forest border region of Wisconsin. Ecology, 32:476-496.

Cysneiros VC, Amorim TA, Mendonça Junior JO, Gaui TD, Moraes JCR, Braz DM, Machado AS (2017) Distribuição diamétrica de espécies da Floresta Ombrófila Densa no Sul do estado do Rio de Janeiro. Revista Pesquisa Florestal Brasileira, 37(89): 10, jan. /marc.

Ferreira LV, Almeida SS, Amaral DD, Parolin P (2005) Riqueza e composição de espécies da floresta de igapó e várzea da Estação Científica Ferreira Penna: subsídios para o plano de manejo da Floresta Nacional de Caxiuanã. Pesquisas, Botânica, Instituto Anchietano de Pesquisas, São Leopoldo, (56):103-116.

Food and Agriculture Organization of the United Nations - FAO (1971) Silvicultural research in the Amazon. Roma: Food and Agriculture Organization of the United Nations, (Technical Report, 3). 192p.

Freitas VCM, Silva CE, Antonio Filho PSS, Arruda AC (2005) Estudo alelopático e antimicrobiano de folhas de Eschweilera sp.e Mabea fistulifera. $29^{a}$ Reunião Anal da Sociedade Brasileira de Química, Águas de Lindóia, São Paulo.

Gama JRV, Botelho SA, Bentes-Gama MM (2002) Composição florística e estrutura da regeneração natural de floresta secundária de várzea baixa no estuário amazônico. Revista Árvore, 26(5):559-566.

Gama JRV, Botelho SA, Bentes-Gama MM, Scolforo JRS (2003) Estrutura e potencial futuro de utilização da regeneração natural de floresta de várzea alta no município de Afuá, estado do Pará. Ciência Florestal, 13(2):71 - 82.

Gama JRV, Souza AL, Martins SV, Souza DR (2005). Comparação entre florestas de várzea e de terra firme do Estado do Pará. Revista Árvore, 29(4): 607-616.

Heinsdijk D, Bastos AM (1963) Inventários florestais na Amazônia. Boletim do Setor de Inventário Florestal, 6:110.

Higuchi N, Jardim FCS, Santos J, Alencar JC (1985) Bacia 3 - Inventário diagnóstico da regeneração natural. Acta Amazonica, 15(1-2):199-233.

Jardim MAG, Vieira ICG (2001) Composição florística e estrutura de uma floresta de várzea do estuário amazônico, Ilha do Combu, Estado do Pará, Brasil. Boletim do Museu Paraense Emilio Goeldi, Série Botânica, 17(2):333-354.

Lima RR, Tourinho MM, Costa JPC da (2000) Várzeas flúvio-marinhas da Amazônia brasileira: características $e$ possibilidades agropecuárias. Belém: FCAP, 341 p.

Lorenzi H (2009) Árvores Brasileiras: manual de identificação e cultivo de plantas arbóreas nativas do Brasil, Nova Odessa: Plantarum, 352p.

Macedo DS, Oliveira Junior PHB, Nogueira ELS, Guedelha C (2007) Produção madeireira, comercialização e o potencial para a certificação florestal nas várzeas: perspectivas para o novo milênio. In: Salomão RP, Terezo EFM, Jardim MAG Manejo florestal nas várzeas: 
oportunidades e desafios. Belém: Museu Paraense Emilio Goeldi, p.139-175.

Mori SA, Prance GT (1990) Taxonomy, ecology, and economic botany of the Brazil nut (Bertholletia excelsa Humb. \& Bonpl.: Lecythidaceae). In. Advances in Economic Botany (8). New York, p.130-150.

Mueller-Dombois D, Ellemberg H (1974) Aims and methods of vegetation ecology. New York: John Wiley $\&$ Sons. 546p.

Oliveira AF (2000) Estrutura genética de populações naturais de Copaifera Langsdorffii Desf. a partir de isoenzimas. Dissertação, Universidade Federal de Lavras. $114 \mathrm{p}$.

Pires JM, Prance GT (1985) The vegetation types of the Brasilian Amazon. In: Prance, G.T, Lovejoy, T.E. (Eds.) Amazonia: Key Environment. Pergamon Press, p.109-145.

Queiroz JAL (2004) Fitossociologia e distribuição diamétrica em floresta de várzea do estuário do rio Amazonas no Estado do Amapá. Dissertação, Universidade Federal do Paraná, Curitiba, 113p.

Queiroz JAL (2008) Estrutura e dinâmica em uma floresta de várzea do rio Amazonas no Estado do Amapá. Tese, Universidade Federal Paraná, Curitiba. 163p.

Renó VF, Novo EML, Almeida Filho R, Suemitsu C (2011) M apeamento da antiga cobertura vegetal de várzea do Baixo Amazonas a partir de imagens históricas (19751981) do Sensor MSS-Landsat. Acta Amazonica, 41(1):47-56.

Seitz RAA (1988) Análise do povoamento: o primeiro passo. Floresta, 18(1-2):4-11.

Silva DAS, Aparicio WCS, Aparicio OS, Batista APB, Matos Filho JR, Lima RB (2011) Estrutura, distribuição espacial e volumetria de Carapa guianensis Aubl. em uma floresta de várzea no estado do Amapá, Brasil. In $5^{\circ}$ Simpósio Latino Americano sobre manejo florestal.

Silva Ribeiro RB, Gama JRV, Martins SV, Moraes A, Santos CAA, Carvalho NA (2013) Estrutura florestal em projeto de assentamento, comunidade são Mateus, município de placas, Pará, brasil. Revista Ceres, 60(5):610-620.

Silva VSM (2006) Manejo de florestas nativas: planejamento, implantação e monitoramento. Universidade Federal do Mato Grosso. Cuiabá, 114p.

Souza LA, Aparício PS, Aparicio WCS, Sotta ED, Guedes MC, Oliveira LPS (2011) Estrutura populacional da espécie Vouacapoua americana Aubl. em floresta de terra firme no estado do Amapá, Brasil. In: $5^{\circ}$ Simpósio latino americano sobre manejo florestal, Santa Maria.

Vale VS, Crespilho RF, Schiavini I (2009) Análise da regeneração natural em uma comunidade vegetal do cerrado no Parque Victório Siquierolli, Uberlândia, MG. Bioscience Journal, 25(1):131-145.

Wittmann F, Junk WJ (2003) Sapling communities in Amazonian white-water forests. Journal of Biogeography, 30(1):1533-1544. 